\title{
Is the 6-minute pegboard and ring test valid to evaluate upper limb function in hospitalized patients with acute exacerbation of COPD?
}

This article was published in the following Dove Press journal: International Journal of COPD

\author{
Rosimeire Marcos \\ Felisberto' \\ Cassia Fabiane de Barros' \\ Kelly Cristina Albanezi \\ Nucci' \\ Andre Luis Pereira de \\ Albuquerque' \\ Elaine Paulin ${ }^{2}$ \\ Christina May Moran de \\ Brito' \\ Wellington Pereira \\ Yamaguti'
}

'Hospital Sírio-Libanês, São Paulo, SP, Brazil; ${ }^{2}$ Universidade do Estado de Santa Catarina (UDESC),

Florianópolis, SC, Brazil

Correspondence: Wellington Pereira Yamaguti

Hospital Sírio-Libanês, Rehabilitation

Center, Dona Adma Jafet Street,

9I - Bela Vista, São Paulo/SP,

Brazil, 0| $308-050$

Tel +55 II 972860465

Email wellington.psyamaguti@hsl.org.br
Background: The 6-minute pegboard and ring test (6-PBRT) is a useful test for assessing the functional capacity of upper limbs in patients with stable COPD. Although 6-PBRT has been validated in stable patients, the possibility of a high floor effect could compromise the validity of the test in the hospital setting. The aim of this study was to verify the convergent validity of 6-PBRT in hospitalized patients with acute exacerbation of COPD (AECOPD).

Methods: A cross-sectional study was conducted in a tertiary hospital. Patients who were hospitalized due to AECOPD and healthy elderly participants, voluntarily recruited from the community, were considered for inclusion. All participants underwent a 6-PBRT. Isokinetic evaluation to measure the strength and endurance of elbow flexors and extensors, handgrip strength (HGS), spirometry testing, the modified Pulmonary Functional Status Dyspnea Questionnaire (PFSDQ-M), the COPD assessment test (CAT), and symptoms of dyspnea and fatigue were all measured as comparisons for convergent validity. Good convergent validity was considered if $>75 \%$ of these hypotheses could be confirmed (correlation coefficient $>0.50$ ).

Results: A total of 17 patients with AECOPD (70.9 \pm 5.1 years and forced expiratory volume in 1 second $\left[\mathrm{FEV}_{1}\right]$ of $41.8 \% \pm 17.9 \%$ of predicted) and 11 healthy elderly subjects were included. The HGS showed a significant strong correlation with 6-PBRT performance $(r=0.70 ; p=0.002)$. The performance in 6-PBRT presented a significant moderate correlation with elbow flexor torque peak $(r=0.52 ; p=0.03)$ and elbow extensor torque peak $(r=0.61 ; p=0.01)$. The total muscular work of the 15 isokinetic contractions of the elbow flexor and extensor muscles showed a significant moderate correlation with the performance in 6-PBRT ( $r=0.59 ; p=0.01$ and $r=0.57$; $p=0.02$, respectively). Concerning the endurance of elbow flexors and extensors, there was a significant moderate correlation with 6-PBRT performance $(r=-0.50 ; p=0.04$ and $r=-0.51$; $p=0.03$, respectively). In relation to the upper-extremity physical activities of daily living (ADLs) assessed by means of PFSDQ-M, there was a significant moderate correlation of 6-PBRT with three domains: influence of dyspnea on ADLs $(r=-0.66 ; p<0.001)$, influence of fatigue on ADLs $(r=-0.60 ; p=0.01)$, and change in ADLs in relation to the period before the disease onset ( $r=-0.51 ; p=0.03)$. The CAT was also correlated with 6-PBRT $(r=-0.51 ; p=0.03)$. Finally, the performance in 6-PBRT showed a significant moderate correlation with the increase in dyspnea $(r=-0.63 ; p=0.01)$ and a strong correlation with the increase in fatigue of upper limbs $(r=-0.76$; $p<0.001)$ in patients with AECOPD. Convergent validity was considered adequate, since $81 \%$ from 16 predefined hypotheses were confirmed. There was no correlation between 6-PBRT and patients' height. The performance in 6-PBRT was worse in patients with AECOPD compared to healthy elderly individuals ( $248.7 \pm 63.0$ vs $361.6 \pm 49.9$ number of moved rings; $p<0.001)$.

Conclusion: The 6-PBRT is valid for the evaluation of functional capacity of upper limbs in hospitalized patients with AECOPD.

Keywords: COPD, exacerbation, skeletal muscle, physical activity, exercise capacity 


\section{Introduction}

Acute exacerbation of COPD (AECOPD) contributes to a substantial decline in peripheral muscle function, compromising exercise tolerance, physical activity level, and activities of daily living (ADLs). ${ }^{1,2}$ Although structural and functional changes are found predominantly in the musculature of the lower limbs, ${ }^{3}$ which are the main responsible factors for the limitation of exercise tolerance, it is recognized that this intolerance may also occur in activities involving the upper limbs, especially those performed without support. ${ }^{4} \mathrm{~A}$ previous study demonstrated that shoulder abduction muscle strength is impaired in patients with COPD when compared to healthy individuals, and this weakness is also proportional to the muscle weakness found in the femoral quadriceps, thus suggesting that patients with COPD have systemic muscle involvement. ${ }^{5}$ In addition to muscle dysfunction, the possible mechanisms related to exercise intolerance involving upper limbs are also associated with increased ventilatory and metabolic demand, occurrence of dynamic hyperinflation, and thoracoabdominal asynchrony during these activities. ${ }^{6,7}$ It is assumed that such changes are responsible for the greater sensation of fatigue and dyspnea and early interruption during simple ADLs of self-care, such as eating, personal hygiene, bathing, washing hair, and getting dressed. ${ }^{8}$

Some tests have been described in the literature for assessing the functional capacity of upper limbs in patients with stable COPD, including the unsupported upper limb exercise test, ${ }^{9}$ grocery shelving task,,$^{10}$ and the 6-minute pegboard and ring test (6-PBRT). ${ }^{11}$ The 6-PBRT is the most commonly used and was first described by Celli et al. ${ }^{12}$ However, its applicability, reproducibility, and sensitivity were demonstrated only more recently in patients with stable COPD and pulmonary arterial hypertension. ${ }^{13,14}$ The relationship between 6-PBRT and parameters of pulmonary function, ${ }^{13,15}$ strength and endurance of upper limbs, ${ }^{16,17}$ incremental upper limb test, ${ }^{16}$ and upper-extremity physical ADLs ${ }^{15}$ has been demonstrated in patients with stable COPD. In addition, 6-PBRT has also been used to show the efficacy of upper limb exercise training programs ${ }^{18}$ since its responsiveness has already been documented. ${ }^{16,19}$ Despite this increasing applicability, to date, there are no studies that have used 6-PBRT in hospitalized patients with AECOPD.

There has been great interest in investigating the feasibility of functional tests in the hospital setting, such as the 6-minute walking test, ${ }^{20}$ time up and go test, ${ }^{21} 6 \mathrm{~m}$ gait speed test, ${ }^{22}$ and step tests. ${ }^{23}$ However, none of these tools are focused on upper limb performance, which is especially relevant to understand the limiting factors of ADLs involving upper limbs in patients with stable COPD. ${ }^{4}$ Considering that upper limb activities are impaired and may present progressive worsening during an AECOPD, it is necessary to investigate the validity and feasibility of tools aimed to evaluate the functional capacity of upper limbs. Although 6-PBRT has been validated in stable patients, the possibility of a high floor effect could compromise the validity of the test in the hospital setting. Therefore, it is unclear whether 6-PBRT could be an alternative for functional evaluation of hospitalized patients with AECOPD. This study aimed at verifying the convergent validity of 6-PBRT patients hospitalized due to AECOPD. Our study was based on COnsensusbased Standards for the selection of health Measurement INstruments (COSMIN) and its checklist. ${ }^{24}$ It was previously hypothesized that the 6-PBRT would positively correlate with muscle $(r \geq 0.70)$ and pulmonary function $(r \geq 0.50)$ and would correlate negatively with upper-extremity physical ADL score ( $r \geq 0.50)$, clinical impact of the disease $(r \geq 0.5)$, and symptoms of dyspnea $(r \geq 0.70)$ and fatigue $(r \geq 0.70$; COSMIN boxes F-4, F-5, and F-6).

\section{Patients and methods Ethics and participants}

The sample was obtained consecutively, and patients of both genders admitted to Hospital Sírio-Libanês for the treatment of AECOPD were recruited. The following inclusion criteria were considered: 1) previous medical diagnosis of COPD before hospitalization; ${ }^{25} 2$ ) exacerbation classified as level II according to the American Thoracic Society (ATS) and European Respiratory Society (ERS);26 3) the absence of cognitive or motor deficit that limited the execution of the tests; 4) the absence of any history of lung or cardiovascular disease previously investigated by the patient's physician; 5) body mass index (BMI) $<35 \mathrm{~kg} / \mathrm{m}^{2}$; and 6) age $<85$ years. Exclusion criteria were as follows: 1) inability to perform evaluations adequately according to methodological description, and 2) cardiorespiratory instability during the tests (severe dyspnea, arrhythmias, angina, elevated heart rate above $80 \%$ of maximal heart rate, and peripheral oxygen saturation $\left(\mathrm{SpO}_{2}\right)<88 \%$ refractory to oxygen supplementation). Healthy elderly individuals matched for age, gender, and BMI were also included in the study, according to the following criteria: 1) not being hospitalized; 2) the absence of airflow obstruction confirmed by spirometry; 3) the absence of prior cardiovascular disease; 4) the absence of cognitive or motor deficits that would limit the performance in tests; 5) $\mathrm{BMI}<35 \mathrm{~kg} / \mathrm{m}^{2}$; and 6) age $<85$ years. The same exclusion criteria described for patients with AECOPD 
were considered. The study was previously approved by the research ethics committee of the Hospital Sírio-Libanês (approval protocol HSL 2014-66), and written informed consent was provided by all participants.

\section{Study design and experimental procedures}

All participants in the current study underwent an evaluation protocol performed on 2 consecutive days. Patients with AECOPD started the protocol when they presented the following minimum clinical criteria: the use of noninvasive ventilation for $<2$ hours per 6-hour period, resting dyspnea $<7$ (very intense) on modified Borg scale, respiratory rate $<25$ incursions/minute, $\mathrm{SpO}_{2}>88 \%$ (considering the use of oxygen supplementation), and the absence of paradoxical respiratory pattern. On the first day of evaluation, all participants underwent pulmonary function test and 6-PBRT. For patients with AECOPD, two specific questionnaires related to pulmonary disease - the modified Pulmonary Functional Status Dyspnea Questionnaire (PFSDQ-M) and the COPD assessment test (CAT) - were also applied. On the second day, all participants underwent isokinetic muscle evaluation of the elbow flexor and extensor muscles and, after 30 minutes, the handgrip strength (HGS) evaluation was performed.

Spirometry testing was performed using a portable spirometer (Koko pulmonary function testing model; nSpire Health Company, Longmont, CO, USA) according to the methods and criteria recommended by ATS and ERS. ${ }^{27}$ Lung function measured at this moment aimed at evidencing the real pulmonary conditions to be correlated with the performance in 6-PBRT. The parameters obtained at this evaluation were not intended to make the diagnosis of COPD. Upper-extremity ADLs were assessed using PFSDQ-M specific to patients with COPD and validated for the Brazilian population. ${ }^{28}$ This questionnaire is composed of three domains: dyspnea influence on ADLs, influence of fatigue on ADLs, and change in ADLs in comparison to the period before the disease onset. ${ }^{29}$ Regarding the first two domains, the patient reported how much dyspnea and fatigue interfere with 10 specific items of ADLs, choosing for each activity a value between 0 and 10, being 0 (no interference), 1-3 (mild interference), 4-6 (moderate interference), 7-9 (severe interference), and 10 (very serious interference). In the third domain, the patient reported how great the impact of the changes in ADLs was when compared to the period before the disease onset, choosing for each activity a value between 0 and 10 , with 0 (as active as always in relation to this activity), 1-3 (small change), 4-6 (moderate change), 7-9 (extreme change), and 10 (do not do this activity anymore). ADLs were standardized according to the instrument and consisted of, for example: 1, brush hair; 2 , raise arms above head; 3 , shower; 4, wash hair; 5 , put on shirt; 6, prepare snack. A partial score was calculated, ranging from 0 to 100 for each of the three domains, totaling a value ranging from 0 to 300 . Higher scores on the scale indicated greater limitation in ADLs. The clinical impact of COPD symptoms was evaluated by means of CAT using a validated version for the Brazilian population. ${ }^{30}$ The instrument is composed of eight items: cough, catarrh, chest tightness, shortness of breath, limitations in home activities, confidence in leaving home, sleep, and energy. For each item, the patient should choose only one response option, whose score ranges from 0 to 5 . The results vary according to the range of the sum of the obtained scores and can be classified as follows in relation to the clinical impact: $6-10$ points, mild; $11-20$, moderate; $21-30$, severe; $31-40$, very severe. ${ }^{31}$ The 6-PBRT was used to evaluate the functional capacity of upper limbs and was performed according to the method described by Zhan et al. ${ }^{13}$ The test consisted of moving the rings from two fixed pins on one level to an other two fixed pins on a higher level on a vertical support. Two pins were positioned at shoulder height and the other two at $20 \mathrm{~cm}$ above shoulder level. A total of 10 rings (weighing $50 \mathrm{~g}$ each) were placed on each lower pin. Participants were asked to use both hands simultaneously and move the rings from the lower level to the upper level. After placing all the rings on the upper level, the participants were asked to move the rings to the lower level and so on. This cycle should be repeated as many times as possible in 6 minutes. The final score was the total number of rings displaced. The test could be interrupted if there was intense dyspnea, fatigue, or any other severe discomfort, and participants were encouraged to retake the test as soon as possible without the timer being paused. The evaluator encouraged participants using standardized phrases every minute during the test following ATS recommendations. ${ }^{32}$ Dyspnea and fatigue scores of upper limbs were obtained using the modified Borg scale before and immediately after the test. Respiratory rate, $\mathrm{SpO}_{2}$, systolic blood pressure, diastolic blood pressure, and heart rate were also measured before and immediately after the test.

The strength, work, and resistance of elbow flexor and extensor muscles of the dominant upper limb were measured using an isokinetic dynamometer (Biodex Multi Joint System III, PRO; Biodex Medical Systems, Inc., Shirley, NY, USA). During the tests, the individual sat upright on the dynamometer chair, with back support, stabilized with belts at the level of the thorax and pelvis. The participants were instructed to maintain the contralateral hand on the thigh, 
the shoulder in neutral position, with $45^{\circ}$ of flexion and $10^{\circ}$ of abduction. The lateral epicondyle of the humerus was aligned with the axis of rotation of the dynamometer. The protocol consisted of a series of 15 repetitions at a constant angular velocity of $90^{\circ} /$ second and a range of motion of $90^{\circ}$. Maximum isokinetic strength was defined as the highest peak torque in this series of 15 repetitions. To determine the isokinetic muscular resistance, the variation of the mean of the peak torque of the first three repetitions was calculated in relation to the mean of the last three repetitions. This variation was expressed in percentage. A higher proportional decline in peak torque over the 15 repetitions indicated lower muscle endurance. ${ }^{33}$ Peripheral muscle strength was also measured by HGS using a manual hydraulic dynamometer (SH 5001; SAEHAN Corporation, Masan, South Korea) respecting the protocol recommended by the American Association of Hand Therapists. ${ }^{34}$ The scales and the other instruments selected to be compared to the 6-PBRT score are widely used in pulmonary rehabilitation programs, and they have demonstrated validity, reliability, and responsiveness (COSMIN boxes F-7 and F-8). ${ }^{29,31,33,34}$

\section{Statistical analyses}

For statistical analyses, the Statistical Package for Social Sciences 17.0 (SPSS Inc., Chicago, IL, USA) was used. Data are presented as mean \pm SD. The Shapiro-Wilk test was used to evaluate the normality of the data. To evaluate the correlation of the performance in 6-PBRT with the strength, work, and isokinetic muscular endurance of elbow flexors and extensors, HGS, domains and subdomains of PFSDQ-M, CAT, dyspnea and fatigue symptoms, forced expiratory volume in 1 second $\left[\mathrm{FEV}_{1}\right]$, and $\mathrm{FVC}$, the Pearson's correlation test (parametric data) or Spearman's correlation test (nonparametric data) were used. The correlation coefficient was interpreted according to the Munro classification system: "little or no correlation" for values between 0 and 0.25 ; "weak correlation" for values between 0.26 and 0.49 ; "moderate correlation" for values between 0.50 and 0.69 ; "strong correlation" for values between 0.70 and 0.89 ; "very strong correlation" for values between 0.90 and 1.00. ${ }^{35}$ Nonparametric Mann-Whitney test was used for comparison between groups, and the nonparametric Wilcoxon test was used to compare the measurements obtained before and after 6-PBRT. According to the COSMIN guidelines, it is possible to perform the sample size calculations for expected correlations among measures in validity studies. ${ }^{24}$ Sample size was calculated expecting to find a strong correlation between 6-PBRT and upper limb muscle function by considering the following assumptions: a 0.7 correlation coefficient, a desired power of 0.8 , and an alpha value of 0.05 . Therefore, the need for 14 patients to be included in the study was determined (COSMIN box F-3). Convergent validity was determined by correlation of the performance in 6-PBRT with the following variables: strength, work and isokinetic endurance of elbow flexors and extensors, HGS, pulmonary function, PFSDQ-M, CAT, and symptoms developed during the test (modified Borg scale; COSMIN box F-10). Some of the constructs of the comparator instruments that were used to assess convergent validity do not assess upper limb function directly. However, all the constructs have a clear relation to the main construct under study, despite not being straightforward. ${ }^{36}$ A total of 16 different hypotheses were formulated about expected magnitudes and directions of correlations between 6-PBRT and all the other instruments. Good convergent validity was considered if $>75 \%$ of these hypotheses could be confirmed (correlation coefficient $>0.50) .{ }^{37}$ Divergent validity was determined by correlating the performance in 6-PBRT with patients' height, considering that it would be hypothesized not to find a significant correlation between these variables. For all tests, $p<0.05$ was considered statistically significant.

\section{Results}

A total of 73 patients diagnosed with COPD and hospitalized for exacerbation of the disease were screened. Of the 32 eligible patients, 15 were excluded because they refused to participate or because they dropped out during the study (Figure 1) (COSMIN box F-9). Thus, 17 patients with AECOPD were included (10 females $-59 \%$ ) with a mean age of $70.9 \pm 5.1$ years and $\mathrm{FEV}_{1}$ of $41.8 \% \pm 17.9 \%$ of predicted. According to the GOLD classification, 35\% of the patients were categorized as GOLD II, 30\% as GOLD III, and 35\% as GOLD IV. A total of 11 healthy elderly individuals were included (six females, $55 \%$ ) with a mean age of $71.0 \pm 8.4$ years and $\mathrm{FEV}_{1}$ of $93.7 \% \pm 6.6 \%$ of predicted. The baseline characteristics are described in Table 1 . There were no missing values on any variables (COSMIN boxes F-1 and F-2).

\section{Convergent validity}

The HGS showed a significant strong correlation with 6-PBRT performance $(r=0.70 ; p=0.002)$ in patients with AECOPD (Figure 2). The performance in 6-PBRT presented a significant moderate correlation with elbow flexor torque peak $(r=0.52 ; p=0.03)$ and elbow extensor torque peak $(r=0.61 ; p=0.01)$. The total muscular work of the 15 isokinetic contractions of the elbow flexor and extensor muscles showed a significant moderate correlation with the performance in 6-PBRT ( $r=0.59 ; p=0.01$ and $r=0.57 ; p=0.02$, respectively). 


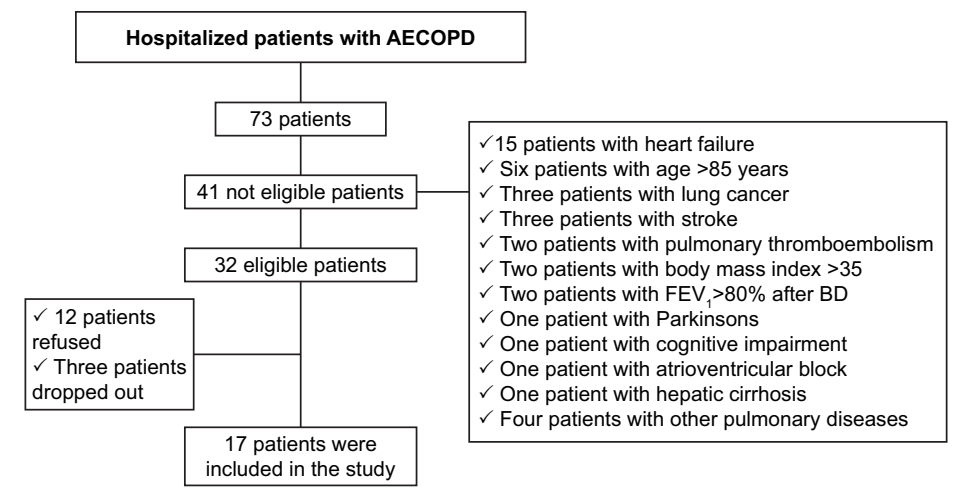

Figure I Screening flowchart for hospitalized patients with AECOPD.

Abbreviations: AECOPD, acute exacerbation of COPD; $\mathrm{BD}$, bronchodilator; $\mathrm{FEV}$, forced expiratory volume in I second.

Concerning the endurance of elbow flexors and extensors, there was a significant moderate correlation with 6-PBRT performance $(r=-0.50 ; p=0.04$ and $r=-0.51 ; p=0.03$, respectively; Figure 3). In general analysis, including patients with AECOPD and healthy elderly participants, a significant strong correlation was observed between 6-PBRT performance and total elbow flexors muscle work $(r=0.73 ; p<0.001$; Figure 4$)$. In relation to the upper-extremity physical ADLs assessed by means of PFSDQ-M, there was a significant moderate correlation of 6-PBRT with three domains: dyspnea influence on ADLs $(r=-0.66 ; p<0.001)$, influence of fatigue on the ADLs $(r=-0.60 ; p=0.01)$, and change in ADLs in relation to the period before the disease onset $(r=-0.51 ; p=0.03)$. The CAT was also correlated with 6-PBRT $(r=-0.51 ; p=0.03)$. Finally, the performance in 6-PBRT showed a significant moderate correlation with the increase in dyspnea $(r=-0.63$;

Table I Characterization of the study participants

\begin{tabular}{|c|c|c|c|}
\hline $\begin{array}{l}\text { Demographic and } \\
\text { anthropometric } \\
\text { characteristics }\end{array}$ & $\begin{array}{l}\text { Patients with } \\
\text { AECOPD } \\
(n=17)\end{array}$ & $\begin{array}{l}\text { Healthy elderly } \\
\text { participants } \\
(n=I I)\end{array}$ & $p$-value \\
\hline Age (years) & $70.9 \pm 5.1$ & $71.0 \pm 8.4$ & 0.93 \\
\hline Gender (F/M) & $10 / 7$ & $6 / 5$ & 0.98 \\
\hline Body mass (kg) & $69.6 \pm 13.7$ & $64.1 \pm 8.4$ & 0.19 \\
\hline Height (m) & $1.66 \pm 0.09$ & $1.60 \pm 0.80$ & 0.09 \\
\hline BMI $\left(\mathrm{kg} / \mathrm{m}^{2}\right)$ & $25.4 \pm 4.7$ & $25.0 \pm 1.1$ & 0.74 \\
\hline GOLD stages (II/III/IV) & $6 / 5 / 6$ & NA & \\
\hline \multicolumn{4}{|l|}{ Pulmonary function } \\
\hline FEV (\% predicted) & $41.8 \pm 17.9$ & $93.7 \pm 6.6$ & $<0.00 I^{*}$ \\
\hline FVC (\% predicted) & $60.9 \pm 19.2$ & $92.6 \pm 7.2$ & $<0.00 I^{*}$ \\
\hline $\mathrm{FEV} / \mathrm{FVC}$ & $0.50 \pm 0.13$ & $0.80 \pm 0.03$ & $<0.00 I^{*}$ \\
\hline VC (\% predicted) & $62.4 \pm 19.5$ & $87.2 \pm 6.7$ & $<0.00 I^{*}$ \\
\hline IC (L) & $1.45 \pm 0.38$ & $2.32 \pm 0.60$ & $<0.00 I^{*}$ \\
\hline
\end{tabular}

Notes: Data are presented as mean \pm SD. FEV $/ / F V C$, the ratio of $F E V_{1}$ to FVC; $\mathrm{n}$, number of individuals. *Significant difference.

Abbreviations: AECOPD, acute exacerbation of COPD; BMI, body mass index; $F$, female; $F E V_{1}$, forced expiratory volume in I second; FVC, forced vital capacity; GOLD, Global Initiative for Chronic Obstructive Lung Disease; IC, inspiratory capacity; M, male; NA, not applicable; VC, vital capacity. $p=0.01)$ and a strong correlation with the increase in fatigue of upper limbs $(r=-0.76 ; p<0.001)$ in patients with AECOPD, measured by the modified Borg scale before and after 6-PBRT, suggesting that patients with poor performance in 6-PBRT develop more dyspnea and fatigue during the test (Figure 5). There was no statistically significant correlation between the performance in 6-PBRT and $\mathrm{FEV}_{1}(p=0.16)$. We found no significant correlation between the performance in 6-PBRT and FVC ( $p=0.16)$. Regarding the pretest inspiratory capacity, there was also no significant correlation with the performance in 6-PBRT $(p=0.22)$. Convergent validity was considered adequate, since 13 predefined hypotheses $(81 \%)$ from a total of 16 were confirmed (correlation coefficients between 6-PBRT and the variables were $>0.50$ ). ${ }^{36}$

\section{Divergent validity}

There was no statistically significant correlation between the performance in 6-PBRT and the height of patients

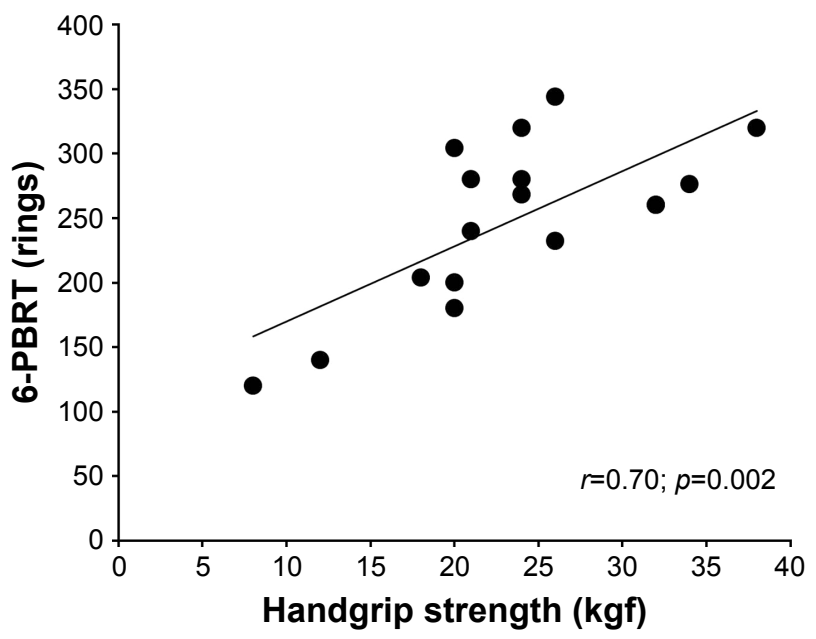

Figure 2 Correlation of manual HGS with performance in 6-PBRT in patients with AECOPD.

Abbreviations: AECOPD, acute exacerbation of COPD; HGS, handgrip strength; 6-PBRT, 6-minute pegboard and ring test. 

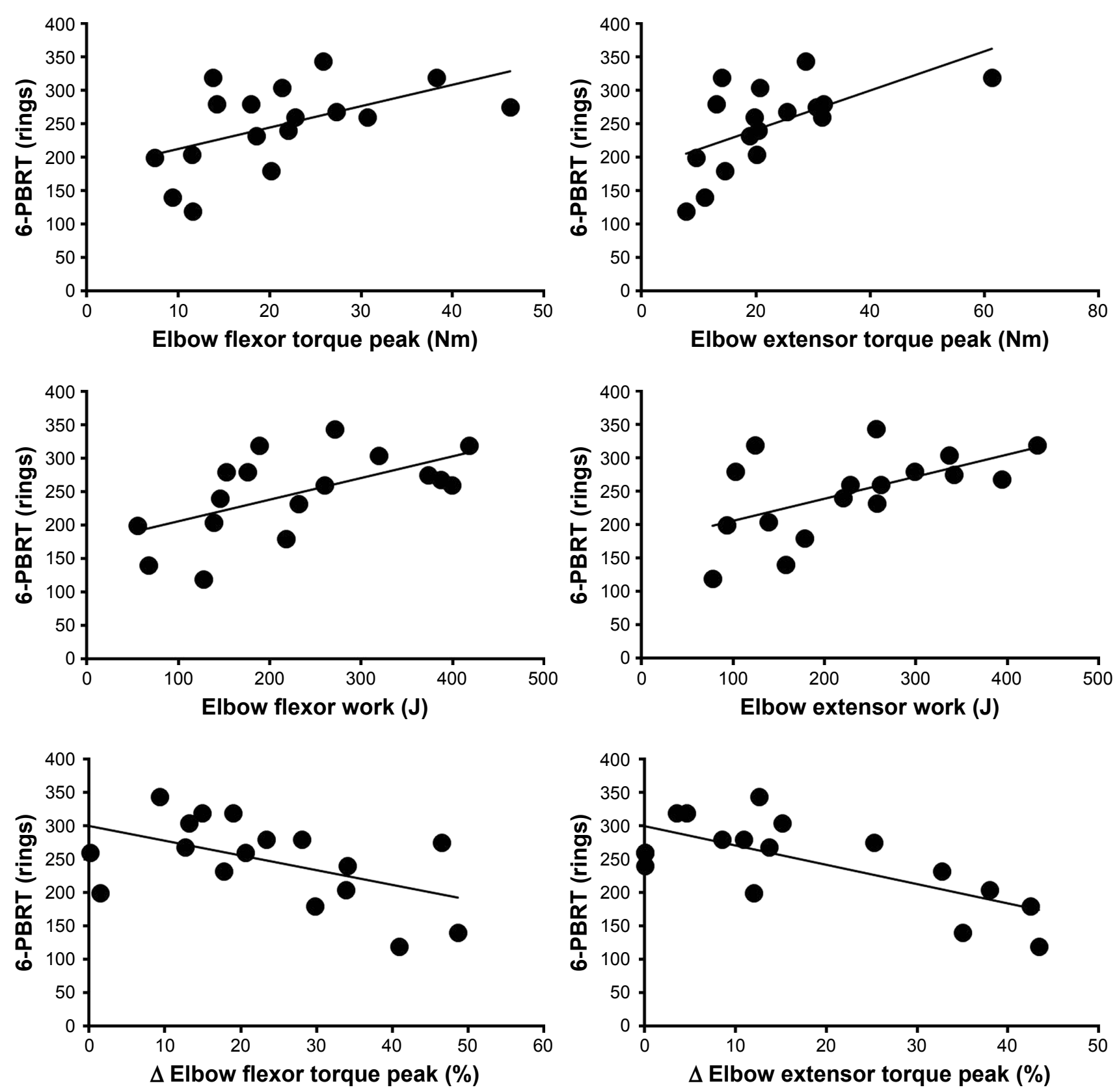

Figure 3 Correlation of 6-PBRT performance with strength, work, and muscular endurance of elbow flexors and elbow extensors in patients with AECOPD. Abbreviations: AECOPD, acute exacerbation of COPD; 6-PBRT, 6-minute pegboard and ring test.

with AECOPD ( $p=0.19$ ), evidencing the divergent validity of 6-PBRT.

Comparison of upper limb muscle function, performance in 6-PBRT, dyspnea, and fatigue among patients with AECOPD and healthy elderly participants Patients with AECOPD presented worse performance in HGS tests and isokinetic muscle evaluation of the strength and endurance of elbow flexors and extensors when compared to healthy elderly individuals (Table 2). The performance in 6-PBRT was also worse in patients (248.7 \pm 63.0 number of moved rings) when compared to healthy elderly individuals, whose mean displacement was $361.6 \pm 49.9$ rings $(p<0.001$; Figure 6). It could be expected that patients with AECOPD might present poor performances in the test and a consequent floor effect might be seen. However, the number of moved rings ranged from 120 to 344 . This wide interval allowed distinguishing patients with different performances, which is desirable to establish the validity of the test. There was 


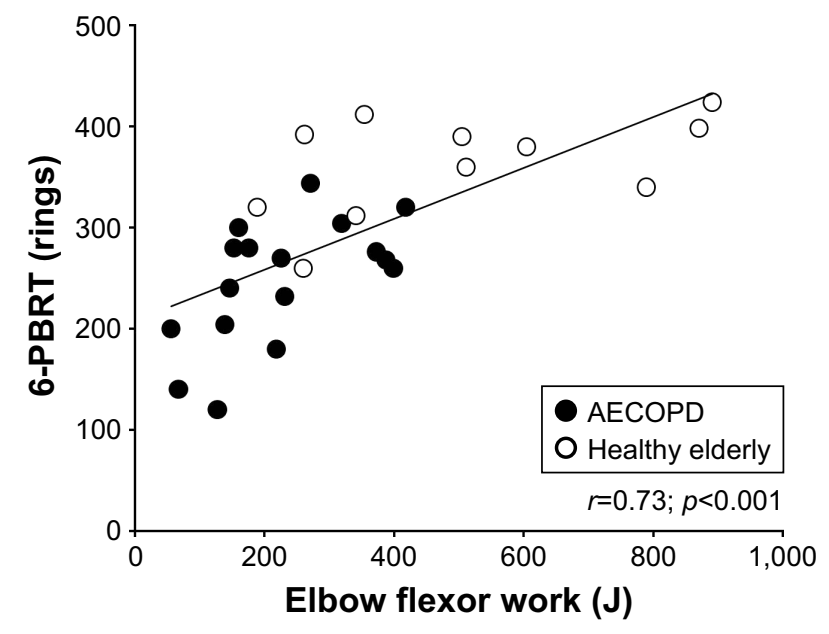

Figure 4 Correlation of 6-PBRT performance with elbow flexor work in patients with AECOPD and healthy elderly participants.

Abbreviations: AECOPD, acute exacerbation of COPD; 6-PBRT, 6-minute pegboard and ring test.

a significant increase in dyspnea (Figure 7A) and fatigue (Figure 7B) during 6-PBRT in patients with AECOPD $(p<0.001)$, and in healthy elderly participants there was a significant increase in fatigue only ( $p=0.002$; Figure 7B). In addition, patients with AECOPD had a greater sensation of dyspnea and fatigue at the end of 6-PBRT when compared to healthy elderly participants (Figure 7A and B; $p<0.001$ ).

\section{Discussion}

The current study aimed to verify the validity of the 6-PBRT for the evaluation of the functional capacity of upper limbs in hospitalized patients with AECOPD, since no test for this purpose has been validated for these patients in the hospital setting. Our results demonstrated the convergent validity of 6-PBRT through the correlation of the performance in the test with parameters of peripheral muscle function, upper-extremity physical ADLs, clinical impact of the disease, and symptoms of dyspnea and fatigue. In addition, it was observed that 6-PBRT was able to differentiate the performance of patients with AECOPD and healthy elderly individuals with similar ages.

There are few studies that have evaluated the convergent validity of 6-PBRT by means of its correlation with parameters of peripheral muscle function in patients with stable COPD. In a previous study, the authors correlated the performance in 6-PBRT with the peripheral muscle strength measured by means of a manual isometric dynamometer. ${ }^{16}$ The muscle groups evaluated were elbow and shoulder flexors and both showed a significant moderate correlation with the performance in 6-PBRT. Although, no previous study correlated performance in 6-PBRT with isokinetic muscle function of the brachial biceps and triceps, Nyberg et $\mathrm{al}^{17}$ found a significant moderate correlation with isokinetic muscle function of shoulder flexors in patients with moderate-to-severe COPD in the stable phase. The authors further suggest that muscular resistance of shoulder flexors appears to have a greater influence on the functional capacity of upper limbs than muscle strength. Only one previous study correlated HGS with 6-PBRT performance in patients with stable COPD in outpatient follow-up, and the results were not statistically significant. ${ }^{15}$

Convergent validity of 6-PBRT in patients with stable COPD, in outpatient follow-up, has also been performed through correlation analysis of performance in 6-PBRT with domains and subdomains of PFSDQ-M. ${ }^{13,15}$ In the first study, a significant moderate correlation was observed in the performance in 6-PBRT with the domain of change in ADLs in relation to the period before the disease onset ( $r=-0.39$; $p=0.04$ ), and with subdomains represented by changes in six ADLs involving upper limbs (1, brush hair; 2, raise arms above head; 3 , shower; 4, wash hair; 5 , put on shirt; 6 , prepare snack). ${ }^{13}$ In the current study, we found similar results, identifying a significant moderate correlation of 6-PBRT performance with the change in ADLs in relation to the period before the disease onset $(r=-0.51 ; p=0.01)$. In addition, a significant moderate correlation was also found in the other two domains of PFSDQ-M: dyspnea $(r=-0.66 ; p<0.001)$ and fatigue ( $r=-0.60 ; p=0.01)$. Our results also agree with the findings of another validation study in which the authors suggest that 6-PBRT may be considered a reference index or potential test for the evaluation of upper-extremity ADLs because it presents a significant moderate correlation with PFSDQ-M subdomains and the level of physical activity of upper limbs measured by means of wrist accelerometers. ${ }^{15}$ Regarding the clinical impact of COPD, the results of the current study suggest that the worse the functional capacity of upper limbs in hospitalized patients with AECOPD, the greater the clinical impact of respiratory symptoms, the limitation of ADLs, selfconfidence, and disposition. This finding was demonstrated by the significant moderate correlation between 6-PBRT performance and CAT. Although no other study has correlated CAT with the performance in 6-PBRT, a previous study has shown a significant correlation between CAT and the distance walked in the 6-minute walking test. ${ }^{30}$

Another important finding of the current study was that the greater the dyspnea and fatigue developed during 6-PBRT, the worse the functional capacity of upper limbs in patients with AECOPD. Despite the absence of other studies demonstrating this correlation, it is already well established in the literature 

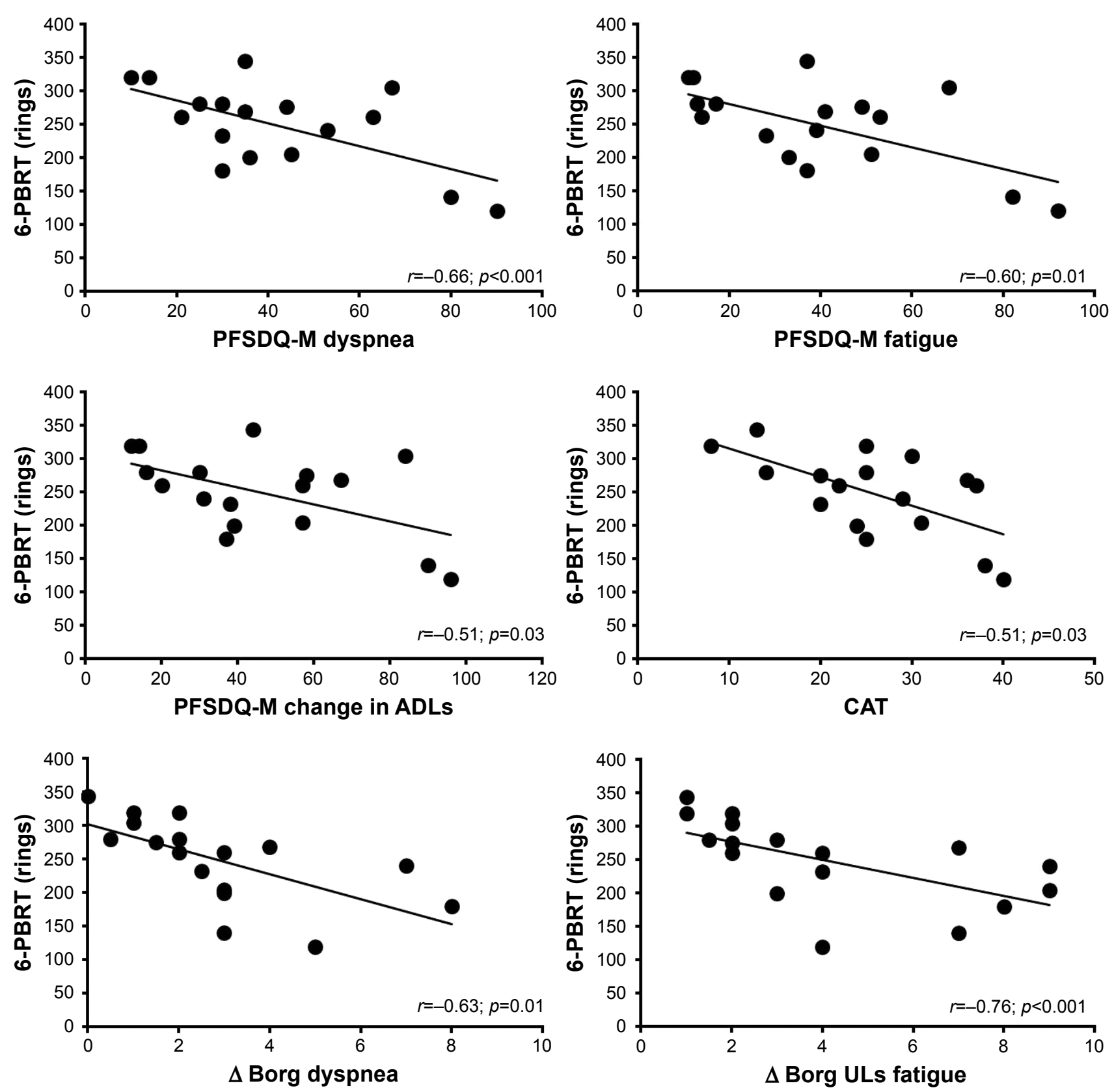

Figure 5 Correlation of 6-PBRT performance with PFSDQ-M domains, CAT, and symptoms in patients with AECOPD.

Abbreviations: ADL, activities of daily living; AECOPD, acute exacerbation of COPD; CAT, COPD assessment test; 6-PBRT, 6-minute pegboard and ring test; PFSDQ-M, modified Pulmonary Functional Status Dyspnea Questionnaire; UL, upper limb.

that the sensation of dyspnea and fatigue may be one of the main limiters of unsupported upper limb exercise tolerance in patients with COPD. ${ }^{38-40}$ The strong correlation found for the perception of fatigue corroborates our findings that also demonstrated the correlation of 6-PBRT with the resistance of the elbow flexor and extensor muscles. Therefore, our results suggest that patients who develop greater sensation of fatigue and present worse muscular function may present worse functional capacity of upper limbs. To the best of our knowledge, there are no other studies published in the literature which have correlated the performance in 6-PBRT with the sensation of dyspnea and fatigue developed during the test.

The absence of correlation between pulmonary function parameters and 6-PBRT performance in our study was not surprising. Only one study demonstrated a moderate significant correlation of 6-PBRT performance with $\mathrm{FEV}_{1}$ and FVC. ${ }^{13}$ One hypothesis for this lack of correlation can be explained by other authors, who suggest that the degree of airflow obstruction and lung volumes and capacities may 
Table 2 Muscle performance of patients with AECOPD and healthy elderly participants

\begin{tabular}{|c|c|c|c|}
\hline $\begin{array}{l}\text { Upper limb muscle } \\
\text { performance }\end{array}$ & $\begin{array}{l}\text { Patients with } \\
\text { AECOPD } \\
(n=17)\end{array}$ & $\begin{array}{l}\text { Healthy elderly } \\
\text { participants } \\
(n=I I)\end{array}$ & $\overline{p \text {-value }}$ \\
\hline HGS (kgf) & $23.5 \pm 7.6$ & $32.1 \pm 8.4$ & $<0.00 I^{*}$ \\
\hline Elbow flexor torque peak ( $\mathrm{Nm})$ & $21.1 \pm 10.3$ & $33.2 \pm 14.8$ & $0.02 *$ \\
\hline Elbow extensor torque peak $(\mathrm{Nm})$ & $22.3 \pm 12.6$ & $36.1 \pm 13.5$ & $<0.00 I^{*}$ \\
\hline Elbow flexor work (J) & $230.9 \pm I 15.4$ & $501.20 \pm 260.62$ & $<0.001 *$ \\
\hline Elbow extensor work (J) & $229.1 \pm 107.4$ & $455.3 \pm 199.2$ & $<0.00 I^{*}$ \\
\hline$\Delta$ Elbow flexor torque peak (\%) & $23.2 \pm 14.4$ & $9.1 \pm 4.8$ & $<0.00 I^{*}$ \\
\hline$\Delta$ Elbow extensor torque peak (\%) & $17.5 \pm 15.4$ & $5.9 \pm 2.6$ & $0.04 *$ \\
\hline
\end{tabular}

Notes: Data are presented as mean \pm SD. n, number of individuals. *Significant difference.

Abbreviations: AECOPD, acute exacerbation of COPD; HGS, handgrip strength.

not be good predictors of exercise capacity assessed by the 6-minute walking test in patients with COPD. ${ }^{41,42}$ In a recent study, it was demonstrated that the distance walked in the 6-minute walking test did not correlate with $\mathrm{FEV}_{1}$. In this study, only femoral quadriceps muscle strength could predict the distance walked. The authors suggest that these results indicate the main influence of peripheral muscle function on submaximal exertion tests. ${ }^{42}$

Previous studies have shown that 6-PBRT is a responsive test for upper limb training in addition to being sensitive and able to differentiate the performance of patients with COPD and healthy elderly individuals from the same age group. ${ }^{13,16}$ Zhan et al ${ }^{13}$ demonstrated that patients with stable COPD presented worse performance in 6-PBRT (impairment of $24 \%$ ) and a greater sensation of dyspnea after the test. Similar results were found in our study, which showed that patients with AECOPD had worse performance in 6-PBRT (impairment of $31 \%$ ) in addition to presenting greater sensation of dyspnea and fatigue immediately after the test

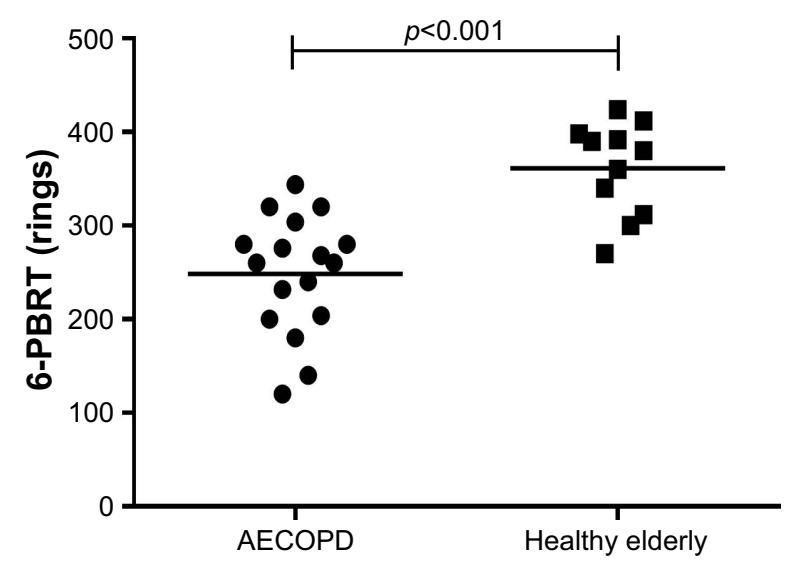

Figure 6 Comparison of 6-PBRT performance among patients with AECOPD and healthy elderly participants.

Abbreviations: AECOPD, acute exacerbation of COPD; 6-PBRT, 6-minute pegboard and ring test. when compared to healthy elderly participants. Therefore, the inclusion of healthy elderly participants aimed at highlighting the discriminatory property of the test. Besides, since there are no reports in the literature on reference equations for 6-PBRT, the inclusion of these participants allowed us to establish a comparative parameter of normality. It is important to note that a shorter test of 2 minutes could be more easily used in the hospital clinical setting and better tolerated by patients with AECOPD. However, unlike other tests focused on lower limb performance, there is no prior description of a 2-minute test for PBRT in the literature.

Divergent validity of 6-PBRT in patients with AECOPD was also demonstrated in this study. We observed the absence of a significant correlation between patients' height and performance in 6-PBRT, thus suggesting that height has no relation to the functional capacity of upper limbs in hospitalized patients with AECOPD. No previous study described in the literature presented the analysis of divergent validity of 6-PBRT in patients with stable COPD or in the acute exacerbation phase.

The main limitation of this study was that the patients did not perform the evaluation of the isokinetic muscle function of the shoulder flexors, since this muscle group plays an important role in the execution of 6-PBRT. The justification for this limitation is due to the fact that patients with AECOPD could present difficulties in performing this test and would not tolerate the isokinetic evaluation of the shoulder joint. Although shoulder flexion is required during 6-PBRT, this movement occurs without any additional resistance and the patient can interrupt the test for limiting reasons at any time. However, during the isokinetic evaluation of the shoulder joint, the patient would need to apply maximum force with constant amplitude against the resistance imposed by the isokinetic dynamometer. Failure to 
A

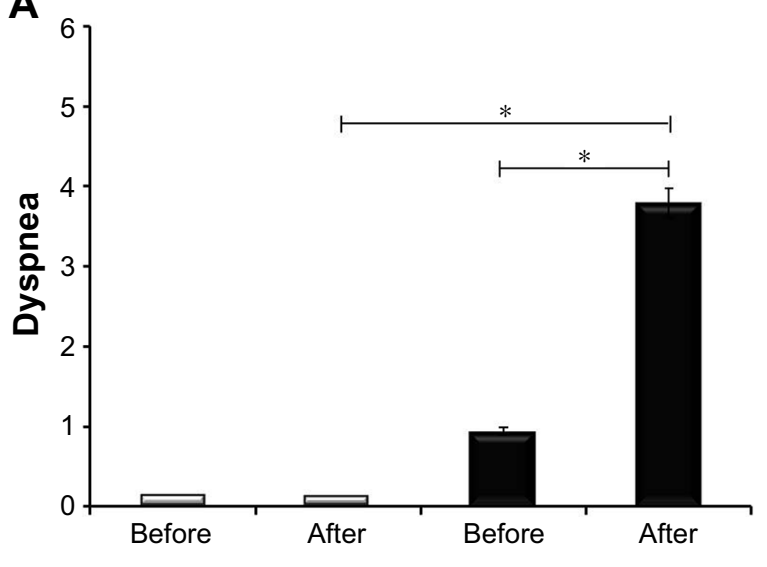

B

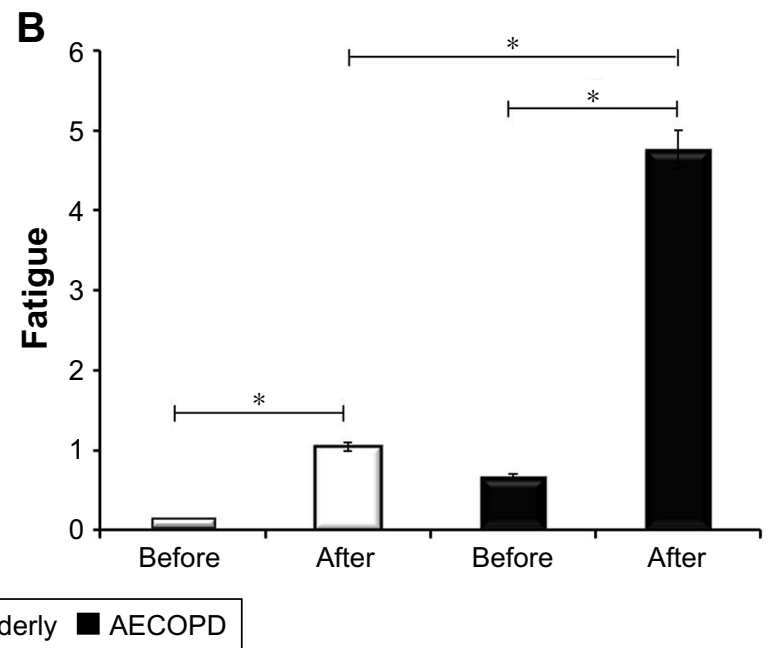

Figure 7 (A) Comparison of the sensation of dyspnea before and after 6-PBRT in patients with AECOPD and healthy elderly participants. (B) Comparison of the sensation of fatigue before and after 6-PBRT in patients with AECOPD and healthy elderly participants. ${ }^{*} p<0.05$

Abbreviations: AECOPD, acute exacerbation of COPD; 6-PBRT, 6-minute pegboard and ring test.

perform isokinetic evaluation properly could compromise the accuracy of measures of muscle strength and endurance. In addition, orthopedic limitations, such as arthrosis, arthritis, and bursitis, frequently found in the elderly population, may be important limitations in performing this evaluation. Another limitation is that our sample size was powered to detect a strong correlation between 6-PBRT and upper limb function. Although we have found a strong correlation of the test with HGS, we only observed moderate correlations with strength, work, and endurance of upper limbs.

In this context, 6-PBRT may be an important tool for assessing the functional capacity of upper limbs to be included within pulmonary rehabilitation programs during the phase of AECOPD in the hospital setting. In addition, the results of the current study suggest that muscle dysfunction, upperextremity physical ADLs, clinical impact of the disease, and symptoms of dyspnea and fatigue are related to the functional capacity of upper limbs. Thus, our study contributes positively to the use of 6-PBRT in hospital practice, contributing to the planning of therapeutic strategies for the rehabilitation of patients with COPD during the acute exacerbation phase.

\section{Conclusion}

We conclude that 6-PBRT is valid for functional capacity assessment of upper limbs in hospitalized patients with AECOPD.

\section{Acknowledgment}

This study was presented in abstract form at the 27th International Congress of the ERS, September 2017, Milan, Italy.

\section{Disclosure}

The authors report no conflicts of interest in this work.

\section{References}

1. Wedzicha JA, Miravitlles M, Hurst JR, et al. Management of COPD exacerbations: a European Respiratory Society/American Thoracic Society guideline. Eur Respir J. 2017;49(3):1600791.

2. Holland AE. Physiotherapy management of acute exacerbations of chronic obstructive pulmonary disease. J Physiother. 2014;60(4):181-188.

3. Abdulai RM, Jellesmark Jensen T, Patel NR, et al. Deterioration of limb muscle function during acute exacerbation of chronic obstructive pulmonary disease. Am J Respir Crit Care Med. 2018;197(4):433-449.

4. Pan L, Guo YZ, Yan JH, Zhang WX, Sun J, Li BW. Does upper extremity exercise improve dyspnea in patients with COPD? A meta-analysis. Respir Med. 2012;106(11):1517-1525.

5. Gosselink R, Troosters T, Decramer M. Distribution of muscle weakness in patients with stable chronic obstructive pulmonary disease. J Cardiopulm Rehabil. 2000;20(6):353-360.

6. Velloso M, Stella SG, Cendon S, Silva AC, Jardim JR. Metabolic and ventilator parameters of four activities of daily living accomplished with arms in COPD patients. Chest. 2003;123(4):1047-1053.

7. Porto EF, Castro AA, Nascimento O, Oliveira RC, Cardoso F, Jardim JR. Modulation of operational lung volumes with the use of salbutamol in COPD patients accomplishing upper limbs exercise tests. Respir Med. 2009;103(2):251-257.

8. Bendixen HJ, Wæhrens EE, Wilcke JT, Sørensen LV. Self-reported quality of ADL task performance among patients with COPD exacerbations. Scand J Occup Ther. 2014;21(4):313-320.

9. Takahashi T, Jenkins SC, Strauss GR, Watson CP, Lake FR. A new unsupported upper limb exercise test for patients with chronic obstructive pulmonary disease. J Cardiopulm Rehabil. 2003;23(6):430-437.

10. Hill CJ, Denehy L, Holland AE, McDonald CF. Measurement of functional activity in chronic obstructive pulmonary disease: the grocery shelving task. J Cardiopulm Rehabil Prev. 2008;28(6):402-409.

11. Bauldoff GS, Rittinger M, Nelson T, Doehrel J, Diaz PT. Feasibility of distractive auditory stimuli on upper extremity training in persons with chronic obstructive pulmonary disease. J Cardiopulm Rehabil. 2005; 25(1):50-55.

12. Celli BR, Rassulo J, Make BJ. Dyssynchronous breathing during arm but not leg exercise in patients with chronic airflow obstruction. NEngl J Med. 1986;314(23):1485-1490. 
13. Zhan S, Cerny FJ, Gibbons WJ, Mador MJ, Wu YW. Development of an unsupported arm exercise test in patients with chronic obstructive pulmonary disease. J Cardiopulm Rehabil. 2006;26(3):180-187; discussion 188-190.

14. Özcan Kahraman B, Özsoy İ, Acar S, et al. [Effect of disease severity on upper extremity muscle strength, exercise capacity, and activities of daily living in individuals with pulmonary arterial hypertension]. Turk Kardiyol Dern Ars. 2017;45(5):434- 440.

15. Takeda K, Kawasaki Y, Yoshida K, et al. The 6-minute pegboard and ring test is correlated with upper extremity activity of daily living in chronic obstructive pulmonary disease. Int J Chron Obstruct Pulmon Dis. 2013;8:347-351

16. Janaudis-Ferreira T, Hill K, Goldstein RS, Wadell K, Brooks D. Relationship and responsiveness of three upper-limb tests in patients with chronic obstructive pulmonary disease. Physiother Can. 2013;65(1): 40-43.

17. Nyberg A, Törnberg A, Wadell K. Correlation between limb muscle endurance, strength, and functional capacity in people with chronic obstructive pulmonary disease. Physiother Can. 2016;68(1):46-53.

18. Nyberg A, Lindström B, Wadell K. Assessing the effect of high-repetitive single limb exercises (HRSLE) on exercise capacity and quality of life in patients with chronic obstructive pulmonary disease (COPD) study protocol for randomized controlled trial. Trials. 2012;13:114.

19. Janaudis-Ferreira T, Hill K, Goldstein RS, et al. Resistance arm training in patients with COPD: a randomized controlled trial. Chest. 2011; 139(1):151-158.

20. Blankenburg T, Guettel A, Busch C, Schuette W. Six-minute walk distance and dyspnoea scores to assess the course of COPD exacerbation in elderly patients. Clin Respir J. 2013;7(3):261-267.

21. Martinez BP, Gomes IB, Oliveira CS, et al. Accuracy of the timed up and go test for predicting sarcopenia in elderly hospitalized patients. Clinics (Sao Paulo). 2015;70(5):369-372.

22. Martinez BP, Batista AK, Ramos IR, et al. Viability of gait speed test in hospitalized elderly patients. J Bras Pneumol. 2016;42(3):196-202.

23. José A, Dal Corso S. Step tests are safe for assessing functional capacity in patients hospitalized with acute lung diseases. J Cardiopulm Rehabil Prev. 2016;36(1):56-61.

24. Mokkink LB, Terwee CB, Knol DL, et al. The COSMIN checklist for evaluating the methodological quality of studies on measurement properties: a clarification of its content. BMC Med Res Methodol. 2010; 10:22.

25. Vestbo J, Hurd SS, Agustí AG, et al. Global strategy for the diagnosis, management, and prevention of chronic obstructive pulmonary disease: GOLD executive summary. Am J Respir Crit Care Med. 2013;187(4): 347-365.

26. Celli BR, MacNee W; ATS/ERS Task Force. Standards for the diagnosis and treatment of patients with COPD: a summary of the ATS/ ERS position paper. Eur Respir J. 2004;23(6):932-946. Erratum in: Eur Respir J. 2006;27(1):242.

27. Miller MR, Hankinson J, Brusasco V, et al. Standardisation of spirometry. Eur Respir J. 2005;26(2):319-338.
28. Kovelis D, Segretti NO, Probst VS, Lareau SC, Brunetto AF, Pitta F. Validation of the modified Pulmonary Functional Status and Dyspnea Questionnaire and the Medical Research Council scale for use in Brazilian patients with chronic obstructive pulmonary disease. J Bras Pneumol. 2008;34(12):1008-1018.

29. Lareau SC, Meek PM, Roos PJ. Development and testing of the modified version of the Pulmonary Functional Status and Dyspnea Questionnaire (PFSDQ-M). Heart Lung. 1998;27(3):159-168.

30. Silva GP, Morano MT, Viana CM, Magalhães CB, Pereira ED Portuguese-language version of the COPD assessment test: validation for use in Brazil. J Bras Pneumol. 2013;39(4):402-408.

31. Jones PW, Harding G, Wiklund I, et al. Tests of the responsiveness of the COPD assessment test following acute exacerbation and pulmonary rehabilitation. Chest. 2012;142(1):134-140

32. Holland AE, Spruit MA, Troosters T, et al. An official European Respiratory Society/American Thoracic Society technical standard: field walking tests in chronic respiratory disease. Eur Respir J. 2014;44(6): 1428-1446.

33. Franssen FM, Broekhuizen R, Janssen PP, Wouters EF, Schols AM. Limb muscle dysfunction in COPD: effects of muscle wasting and exercise training. Med Sci Sports Exerc. 2005;37(1):2-9.

34. Fess EE. Grip strength. In: Casanova JS, editor. Clinical Assessment Recommendations. 2nd ed. Chicago: American Society of Hand Therapists; 1992:41-45

35. Munro BH. Statistical Methods for Health Care Research. 3rd ed. Philadelphia, PA: Lippincott Williams \& Wilkins; 1997.

36. Abma IL, Rovers M, van der Wees PJ. Appraising convergent validity of patient-reported outcome measures in systematic reviews: constructing hypotheses and interpreting outcomes. BMC Res Notes. 2016; 9:226.

37. Scholtes VA, Terwee CB, Poolman RW. What makes a measurement instrument valid and reliable? Injury. 2011;42(3):236-240.

38. Costi S, Crisafulli E, Degli Antoni F, Beneventi C, Fabbri LM, Clini EM. Effects of unsupported upper extremity exercise training in patients with COPD: a randomized clinical trial. Chest. 2009;136(2):387-395.

39. de Souza GF, Castro AA, Velloso M, Silva CR, Jardim JR. Lactic acid levels in patients with chronic obstructive pulmonary disease accomplishing unsupported arm exercises. Chron Respir Dis. 2010;7(2): 75-82

40. McKeough ZJ, Bye PT, Alison JA. Arm exercise training in chronic obstructive pulmonary disease: a randomised controlled trial. Chron Respir Dis. 2012;9(3):153-162.

41. Teixeira PJ, Costa CC, Berton DC, Versa G, Bertoletti O, Canterle DB. [Six-minute walk work is not correlated to the degree of airflow obstruction in patients with Chronic Obstructive Pulmonary Disease (COPD)]. Rev Port Pneumol. 2006;12(3):241-254.

42. Leite Rodrigues S, Melo E, Silva CA, Lima T, de Assis Viegas CA, Palmeira Rodrigues M, Almeida Ribeiro F. The influence of lung function and muscular strength on the functional capacity of chronic obstructive pulmonary disease patients. Rev Port Pneumol. 2009;15(2): 199-214.
International Journal of COPD

\section{Publish your work in this journal}

The International Journal of COPD is an international, peer-reviewed journal of therapeutics and pharmacology focusing on concise rapid reporting of clinical studies and reviews in COPD. Special focus is given to the pathophysiological processes underlying the disease, intervention programs, patient focused education, and self management protocols.

\section{Dovepress}

This journal is indexed on PubMed Central, MedLine and CAS. The manuscript management system is completely online and includes a very quick and fair peer-review system, which is all easy to use. Visi http://www.dovepress.com/testimonials.php to read real quotes from published authors. 